\title{
A correlation-energy density functional for multideterminantal wavefunctions
}

\author{
By BURKHARD MIEHLICH $\dagger$, HERMANN STOLL \\ Institut für Theoretische Chemie, Universität Stuttgart, D-70550 Stuttgart, Germany \\ and ANDREAS SAVIN \\ Laboratoire de Chimie Théorique, Université Pierre et Marie Curie, \\ F-75252 Paris, France
}

(Received 12 December 1996; accepted 2 February 1997)

\begin{abstract}
A density functional for dynamical correlation, to be used in connection with wavefunctions of the complete-active-space self-consistent field type, is described, and first applications to the series of two- and four-electron atomic ions as well as to the $\mathrm{H}_{2}$ potential curve are given.
\end{abstract}

\section{Introduction}

Density functional theory (DFT) has been very successful for both an economic and an accurate description of electronic structure in atomic, molecular and solid-state physics (for example [1]); electron correlation effects are implicitly included while formally retaining an independent-particle Hartree-Fock (HF)-like picture. The HF picture, on the other hand, is just the starting point for an explicit electron correlation treatment with the wavefunction-based quantum-chemical ab initio methods (for example [2]); in contrast with DFT, a systematic improvement towards the exact results is possible, but at the expense of a heavy computational burden caused by long configuration interaction (CI) expansions. However, long CI expansions are usually related to dynamical correlation effects which have to do with the short-range description of the electron correlation hole (electron-electron cusp), while static (longrange) correlation effects caused by near-degeneracies of ground and low-lying excited states of the same symmetry very often can efficiently be covered by quite short optimized expansions (of the multiconfiguration self-consistent field (MCSCF) or complete-active-space self-consistent field (CASSCF) type). Since deficiencies of DFT, if any, are likely to occur just with the description of near-degeneracies which are special to individual molecules and not easily covered by a universal functional, the idea of a CI-DFT coupling combining the advantages of the two approaches seems to be rewarding.

$\dagger$ Present address; Institut für Organische Chemie der Universität, D-70550 Stuttgart, Germany.
In fact, several attempts towards this aim have been reported in literature (see [3] for a review up to 1990). There are two main problems: first, a proper separation between CI and DFT contributions is needed, so that double counting of correlation energy is avoided, that is new density functionals have to be derived which, while transforming into usual DFT correlation-energy functionals for a single-determinant (self-consistent field $(\mathrm{SCF})$ ) reference, lead to vanishing DFT contributions in the limit of complete CI calculations. Secondly, the input quantities for the density functionals have to be modified; individual electron densities $\rho_{\alpha}$ and $\rho_{\beta}$ for the $\alpha$ and $\beta$ spin systems should no longer be used with multideterminant reference wavefunctions, since spin degeneracies of such wavefunctions are difficult to describe with a functional explicitly depending on the spin polarization $\zeta=\left(\rho_{\alpha}-\rho_{\beta}\right) /\left(\rho_{\alpha}+\rho_{\beta}\right)$. The second problem, that is the generalization of $\zeta$ to multideterminant cases, has recently been solved by using the on-top two-particle density $\pi(\mathbf{r}, \mathbf{r})$ as DFT input instead of $\zeta$ $[4,5]$ (see also [6]). However, the situation with regard to the first problem, that is the additional DFT parameter needed to account for the amount of explicitly calculated (CI) correlation energy, is still far from a universally accepted solution and, in fact, several variants are possible here. Such a parameter can either be introduced into the Hamiltonian, for example by splitting the interelectronic interaction $1 / r_{i j}$ into a short- and a long-range part, with only the former treated by DFT [7, 8] or it may be related to the wavefunction, for example by splitting the natural-orbital space into strongly and weakly occupied members, with only the former used for the explicitly correlated reference wavefunction [9] 
Anyhow, it is important for the control parameter to satisfy the following theoretical requirements.

(1) It should have a physical meaning not only for the system under consideration but also for the electron gas, in order to provide a simple means of setting up the required modified density functional.

(2) It should define a proper separation between static and dynamic correlation, for every density, without artefacts (such as steps in the potential curve) in the reference CI calculation.

With global parameters (e.g. when using a screening parameter for $\left.1 / r_{i j}\right)$, the first of the two requirements can certainly be met; however, difficulties may arise with the second.

It is the aim of the present paper to look for a local parameter to be used in connection with standard quantum-chemical CASSCF calculations which satisfies both of the above requirements. It is introduced in section 2, and first applications, to the correlation energies of two- and four-electron atoms or ions as well as to the $\mathrm{H}_{2}$ molecule, are described in section 3; a summary is given in section 4. (A preliminary account of some aspects of the present work has been published in [10]).

\section{Theory}

Let $\Phi$ be a many-electron wavefunction of the MCSCF type. Let us further assume that near-degeneracy effects are fully accounted for in $\Phi$ by including all configurations of a given spatial and spin symmetry which can be generated within a chosen number of (near-degenerate) one-electron orbitals $\left\{\phi_{i}^{a}\right\}$ defining the active space. Usually, in CASSCF calculations, there are additional orbitals $\left\{\phi_{i}^{c}\right\}$ lower in energy which are kept doubly occupied, but we assume the contribution of excitations from these orbitals to the active space either to be unimportant or to be covered explicitly by some kind of internal CI. We invoke use of DFT only for correlation effects involving excitations into the external space $\left\{\phi_{i}^{v}\right\}$ orthogonal to $\left\{\phi_{i}^{c}\right\},\left\{\phi_{i}^{a}\right\}$.

Current DFT approximations (local-density approximation (LDA) and generalized gradient approximation (GGA)) rely on local properties of a given system as input parameters, that is the total electron density at some space point $\mathbf{r}$ :

$\rho(\mathbf{r})=N \int\left|\Phi\left(\mathbf{r} \sigma_{1}, \mathbf{r}_{2} \sigma_{2}, \ldots, \mathbf{r}_{N} \sigma_{N}\right)\right|^{2} \mathrm{~d} \sigma_{1} \mathrm{~d} \mathbf{r}_{2} \mathrm{~d} \sigma_{2} \cdots \mathrm{d} \mathbf{r}_{N} \mathrm{~d} \sigma_{N}$,

and its spin polarization $\zeta(\mathbf{r})=\left[\rho_{\alpha}(\mathbf{r})-\rho_{\beta}(\mathbf{r})\right] \rho(\mathbf{r})$ (where $\rho_{\alpha}$ and $\rho_{\beta}$ are the partial densities of the two spin systems), as well as gradients of $\rho$ and $\zeta$ at the same space point. The (exchange-)correlation energy $\left(\varepsilon_{\mathrm{c}}\right.$ or $\left.\varepsilon_{\mathrm{xc}}\right)$ per particle is derived, as a function of these parameters, with the help of model-system (usually electron-gas) data.

Our approach here is similar but differs in two points: firstly, we replace the set $\{\rho(\mathbf{r}), \zeta(\mathbf{r})\}$ of input parameters by $\{\rho(\mathbf{r}), \pi(\mathbf{r}, \mathbf{r})\}$, with the on-top two-particle density

$$
\begin{aligned}
\tau(\mathbf{r}, \mathbf{r})= & N(N-1) \int \mid \Phi\left(\mathbf{r} \sigma_{1}, \mathbf{r} \sigma_{2}, \mathbf{r}_{3} \sigma_{3}, \ldots,\left.\mathbf{r}_{N} \sigma_{N}\right|^{2}\right. \\
& \times \mathrm{d} \sigma_{1} \mathrm{~d} \sigma_{2} \mathrm{dr}_{3} \mathrm{~d} \sigma_{3} \cdots \mathrm{d} \mathbf{r}_{N} \mathrm{~d} \sigma_{N},
\end{aligned}
$$

since we deal with multideterminant reference wavefunctions for which $\zeta$ is no longer a valid description $[4,5]$ If our model system for generating the correlation-energy density functional were still the electron gas with SCF reference wavefunction, the relation

$$
\zeta(\mathbf{r})=\left(1-2 \frac{\pi(\mathbf{r}, \mathbf{r})}{\rho(\mathbf{r})^{2}}\right)^{1 / 2}
$$

valid for the model system would allow for an easy transformation to existing functionals $\varepsilon_{\mathrm{c}}(\rho, \zeta)$. However, and that is the second point, our model system cannot be the same any longer; we may still use the electron gas, but its reference wavefunction has to be changed. A suitable model for our molecular CASSCF wavefunction would be an electron-gas wavefunction already including part of the correlation effects by means of excitations into low-lying states $\exp (\mathrm{ik} \cdot \mathbf{r})$ above the Fermi level $k_{\mathrm{F}}\left(k_{\mathrm{F}}<|\mathbf{k}|<k_{\mathrm{g}}\right)$. A point-by-point correspondence can be established by equating the (diagonal of the) projection matrices onto the occupied space in the two cases:

$$
\frac{1}{(2 \pi)^{3}} \int_{|\mathbf{k}|<k_{\mathrm{g}}} \mathrm{d} \mathbf{k}(\exp (\mathrm{ik} \cdot \mathbf{r}))^{*} \exp (\mathrm{ik} \cdot \mathbf{r})=\sum_{;, j, a}^{s \in c} \phi_{i}^{s *}(\mathbf{r}) \phi_{i}^{s}(\mathbf{r})
$$

which immediately leads to

$$
\frac{k_{\mathrm{g}}}{k_{\mathrm{F}}}=\left(\sum_{i, s}^{s \in c, a} \frac{2\left|\phi_{i}^{s}(\mathbf{r})\right|^{2}}{\rho(\mathbf{r})}\right)^{1 / 3} .
$$

Thus the extended set of input parameters for generating $\varepsilon_{\mathrm{c}}$ becomes $\left\{\rho(\mathbf{r}), \pi(\mathbf{r}, \mathbf{r}), k_{\mathrm{g}} / k_{\mathrm{F}}\right\}$, according to equations (1), (2) and (5). Obviously, in the limiting case of a closed-shell SCF, we have $\pi=\rho^{2} / 2$ and $k_{\mathrm{g}} / k_{\mathrm{F}}=1$; using equation (3), our modified $\varepsilon_{\mathrm{c}}$ to be discussed presently must transform into one of the currently available DFT approximations.

In order to construct the new $\varepsilon_{\mathrm{c}}$, we start from a calculation described in [9] where results have been obtained for the correlation energy of the (paramagnetic) electron gas, $\hat{\varepsilon}_{\mathrm{c}}\left(r_{\mathrm{s}}, k_{\mathrm{g}} / k_{\mathrm{F}}\right)$, with $r_{\mathrm{s}}=(3 / 4 \pi \rho)^{1 / 3}$, when excitations are restricted to $|\mathbf{k}|<k_{\mathrm{g}}$. For our purposes, 
Figure 1. Comparison of the analytical fit for $\varphi\left(r_{\mathrm{s}}, k\right)$ with the original results of [9] $\left(r_{\mathrm{s}}\right.$ values in atomic units.)

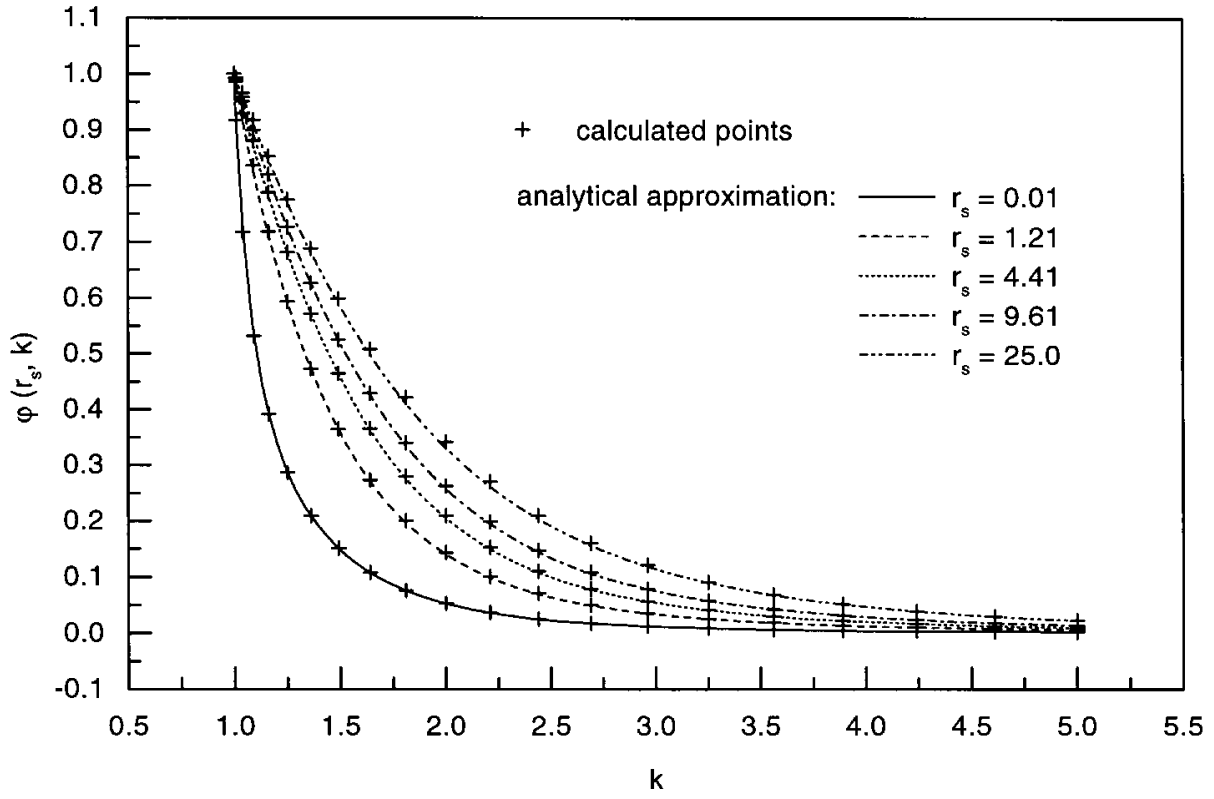

Table 1. Coefficients $b_{m n}$ in the analytical representations of $\varphi\left(r_{\mathrm{s}}, k\right)$, cf. text.

\begin{tabular}{cccrrr}
\hline & \multicolumn{5}{c}{$b_{m n} / \mathrm{au}$} \\
\cline { 2 - 6 }$m$ & $n=1$ & $n=2$ & \multicolumn{1}{c}{$n=3$} & \multicolumn{1}{c}{$n=4$} & $n=5$ \\
\hline 1 & $-0 \cdot 2207193 \times 10^{1}$ & $0 \cdot 6807648 \times 10^{1}$ & $-0 \cdot 6386316 \times 10^{1}$ & $0 \cdot 2860522 \times 10^{1}$ & $-0 \cdot 7466076 \times 10^{-1}$ \\
2 & $0 \cdot 1128469 \times 10^{1}$ & $-0 \cdot 2535669 \times 10^{1}$ & $0 \cdot 2432821 \times 10^{1}$ & $-0 \cdot 1064058 \times 10^{1}$ & $0 \cdot 3843687 \times 10^{-1}$ \\
3 & $-0 \cdot 2475593 \times 10^{0}$ & $0 \cdot 4243142 \times 10^{0}$ & $-0 \cdot 2565175 \times 10^{0}$ & $0 \cdot 8294749 \times 10^{-1}$ & $-0 \cdot 3184296 \times 10^{-2}$ \\
4 & $0 \cdot 8616560 \times 10^{-1}$ & $-0 \cdot 1715714 \times 10^{0}$ & $0 \cdot 1067547 \times 10^{0}$ & $-0 \cdot 2392882 \times 10^{-1}$ & $0 \cdot 2579856 \times 10^{-2}$ \\
5 & $-0 \cdot 6500077 \times 10^{-2}$ & $0 \cdot 1714085 \times 10^{-1}$ & $-0 \cdot 1462187 \times 10^{-1}$ & $0 \cdot 4423830 \times 10^{-2}$ & $-0 \cdot 4427570 \times 10^{-3}$ \\
6 & $-0 \cdot 2491486 \times 10^{-2}$ & $0 \cdot 5321373 \times 10^{-2}$ & $-0 \cdot 3704699 \times 10^{-2}$ & $0 \cdot 9700054 \times 10^{-3}$ & $-0 \cdot 9518308 \times 10^{-4}$ \\
\hline
\end{tabular}

we fitted these results by an analytical expression as follows:

$$
\begin{aligned}
\varphi\left(r_{\mathrm{s}}, k\right) & :=1-\frac{\hat{\varepsilon}_{\mathrm{c}}\left(r_{s}, k\right)}{\hat{\varepsilon}_{\mathrm{c}}\left(r_{s}, \infty\right)} \\
& =\left(\sum_{m n} b_{m n} x^{m-1} k^{n-1}\right)^{-1}, \\
k & =\frac{k_{\mathrm{g}}}{k_{\mathrm{F}}}, \quad x=\ln r_{\mathrm{s}} .
\end{aligned}
$$

$\varphi\left(r_{\mathrm{s}}, k\right)$ describes the reduction in the electron-gas correlation energy when the effect of low-lying excitations is left out, that is just the reduction encountered when part of the correlation energy is calculated separately such as in a CASSCF. (From its definition, $\varphi \rightarrow 1$ for $k_{\mathrm{g}} \rightarrow k_{\mathrm{F}}$, and $\varphi \rightarrow 0$ for $k_{\mathrm{g}} \rightarrow \infty$.) Numerical data for the coefficients $b_{m n}$ are listed in table 1 [11] Plots of $\varphi\left(r_{\mathrm{s}}, k\right)$ for different values of $r_{\mathrm{s}}$, including a comparison with the original data used for fitting, are shown in figure 1 .
As already implied by equation (6), we do not use $\hat{\varepsilon}_{\mathrm{c}}\left(r_{\mathrm{s}}, k\right)$ directly, but only in the form of a correction factor $\varphi\left(r_{\mathrm{s}}, k\right)$. This is done for two reasons.

(1) The values for $\hat{\varepsilon}_{\mathrm{c}}\left(r_{\mathrm{s}}, \infty\right)$ are not as accurate as Ceperley and Alder's quantum Monte Carlo (QMC) values [12] on which current LDA functionals are based.

(2) No values are available for $\hat{\varepsilon}_{\mathrm{c}}$ in the spin-polarized case $\zeta \neq 0$.

Thus, we adopt the following approximation for the new density functional:

$$
\hat{\varepsilon}_{\mathrm{c}}\left(\rho, \nabla \rho, \pi, \frac{k_{\mathrm{g}}}{k_{\mathrm{F}}}\right)=\varphi\left(r_{\mathrm{s}}(\rho), \frac{k_{\mathrm{g}}}{k_{\mathrm{F}}}\right) \varepsilon_{\mathrm{c}}(\rho, \nabla \rho, \zeta(\pi)),
$$

where $\varepsilon_{\mathrm{c}}(\rho, \nabla \rho, \zeta)$ is one of the popular correlationenergy density functionals available (e.g. LDA in the parametrization of Vosko et al. [13] or the GGA in the parametrization of Perdew et al. [14]), and $\zeta(\pi)$ is used in its SCF approximation, equation (3). For CASSCF $\pi$ values which are outside the range of the 
single-determinant pair density $\left(\pi<0\right.$ or $\left.\pi>\rho^{2} / 2\right)$ either an extrapolation of $\varepsilon_{\mathrm{c}}$ to imaginary values of $\zeta$ [4] or a redefinition of $\pi\left(\pi=0\right.$ or $\left.\pi=\rho^{2} / 2\right)$ is possible; the results of section 3 refer to the first possibility.

Let us finally proceed now to a concise formulation of the density-functional scheme underlying our approach. Following similar lines as Levy [15] we can write a universal density functional in the form

$$
F[\rho, \pi]=\min _{\psi_{\rho, \pi}}\left\langle\psi_{\rho, \pi}\left|T+V_{\mathrm{ee}}\right| \psi_{\rho, \pi}\right\rangle,
$$

where $\psi_{\rho, \pi}$ is a wavefunction yielding a given density $\rho$ and on-top two-particle density $\pi$, and $T$ and $V_{\text {ee }}$ are the operators of kinetic energy and electron-electron interaction respectively. In our scheme, $F[\rho, \pi]$ is partitioned into

$$
F_{\mathrm{CAS}}[\rho, \pi]+\int \rho \hat{\varepsilon}_{\mathrm{c}}\left(\rho, \nabla \rho, \pi, \frac{k_{\mathrm{g}}}{k_{\mathrm{F}}}\right) \mathrm{d} \mathbf{r},
$$

where $F_{\mathrm{CAS}}$ has the same form as $F[\rho, \pi]$ but with $\psi_{\rho, \pi}$ restricted to be a CASSCF wavefunction for a given active-space size. The second term in equation (9) has to be considered as an approximation for $F[\rho, \pi]$ $F_{\mathrm{CAS}}[\rho, \pi]$ and the parameter $k_{\mathrm{g}} / k_{\mathrm{F}}$ appearing in the integral is connected to $\psi_{\rho, \pi}$ and its active orbitals via equation (5).

The new functional equations (7) and (9) should remedy a major part of the errors previously found for correlation-energy density functionals built upon SCF reference wavefunctions (for example [7, 16, 17]), since the unphysical long-range behaviour of the non-local exchange with a single-determinant wavefunction for bond-breaking processes for example (which cannot be easily corrected for by a local-density functional) is no longer present in the CAS wavefunction.

As with other density functionals, equation (9) supplemented by $\int \rho V_{\text {ne }} \mathrm{dr}$, where $V_{\text {ne }}$ is the external potential, should be subjected to a minimization with respect to $\rho$. However, the self-consistency effect of a correlation-energy density functional on a SCF wavefunction has been shown to be small [7] Thus, in our first applications to be described in the next section, we perform energy minimization at the CASSCF level only and account for the influence of $\hat{\hat{\varepsilon}}_{\mathrm{c}}$ using the CAS density without further density variation.

\section{Results}

\subsection{The He isoelectronic series}

LDA results for correlation energies of the two-electron $X^{(Z-2)+}$ ions $(X=\mathrm{H}, \mathrm{He}, \mathrm{Li}, \ldots)$ are known to exhibit a qualitatively incorrect trend $\left(E_{\mathrm{c}}^{\mathrm{LDA}} \rightarrow \infty\right.$, while $E_{\mathrm{c}}^{\exp } \rightarrow$ constant) $[18,19]$ It has to be seen how well this incorrect behaviour can be remedied when replacing the SCF reference for density functional by CASSCF wavefunctions of increasing complexity.
In our calculations, we used large uncontracted $10 \mathrm{~s} 6 \mathrm{p} 4 \mathrm{~d}\left(\mathrm{H}^{-}: 10 \mathrm{~s} 8 \mathrm{p} 5 \mathrm{~d}\right)$ basis sets in order to exclude finite-basis artefacts as far as possible. The s functions were taken from van Duijneveldt [20] and Poirier et al. [21] supplemented by additional diffuse exponents $0 \cdot 029$ and 0.012 in the case of $\mathrm{H}^{-}$. The $\mathrm{p}, \mathrm{d}$ polarization sets were chosen to be even-tempered (from [22] for $\mathrm{H}^{-}$ supplemented by diffuse $\mathrm{p}(\mathrm{d})$ exponents $0 \cdot 04,0.016$ $(0 \cdot 104)$; sets with centre 1.5 and scaling factors 2.5 in the case of $\mathrm{He}$; the latter sets rescaled according to $\left(\zeta / \zeta_{\mathrm{He}}\right)^{2}$ (from [21] p. 89) for $\mathrm{Li}^{+}-\mathrm{Ne}^{8+}$ ). CASSCF calculations with increasing size of the active space (1s, 2s, 2s1p, 3s1p, 3s $2 \mathrm{p}, 3 \mathrm{~s} 2 \mathrm{p} 1 \mathrm{~d})$ were performed with the program package Molpro [23-25] One- and twoparticle densities $\rho$ and $\pi$ from these calculations were taken as input for a gradient-corrected correlationenergy density functional (LDA part from [13] and GGA from [14]); the spin polarization was determined from $\pi$ according to equation (3), and an additional active-space-dependent factor was introduced according to equations (5)-(7).

Figure 2 shows deviations from 'experimental' correlation energies, as a function of the active-space size, for both CASSCF-only and CASSCF-DFT calculations. While SCF errors are $40-50 \mathrm{mH}$ for all the ions, and SCF-DFT is still off by about $40 \mathrm{mH}$ for the heaviest ion considered $\left(\mathrm{Ne}^{8+}\right)$, a significant improvement is obtained when enlarging the active space; at the CASSCF level, this improvement is only moderately rapid, since the missing correlation is of dynamical nature mainly, but with CASSCF-DFT accuracies of less than 15 and less than $5 \mathrm{mH}$ are reached already with $2 \mathrm{~s}$ and $2 \mathrm{~s} 1 \mathrm{p}$ spaces respectively.

Figure 3 shows the $Z$ dependence of the CASSCFDFT correlation energies in comparison with the experimental curve. It is seen that, already with the 2 s active space, substantially better results are obtained, but the incorrect $\ln Z$ dependence still persists. However, with the $2 \mathrm{~s} 1 \mathrm{p}$ space, the theoretical curve is virtually parallel to the experimental curve.

In order to discuss the influence of the quality of the density functional on our results, we compare in figure 4 GGA values with LDA values. As expected, the gradient correction is essential, although its influence decreases with increasing size of the active space.

Finally, in figure 5 we demonstrate the effect of our using the pair density $\pi$ (instead of $\zeta$ ) as basic DFT input parameter, besides the one-particle density $\rho$. At the SCF-DFT level, this is no issue, of course, per definition (cf. equation (3)); a small but systematic improvement is found, however, with larger active spaces when $\zeta$ is evaluated using the actual pair densities of the ions instead of adopting the usual closed-shell value of $\zeta=0$. 


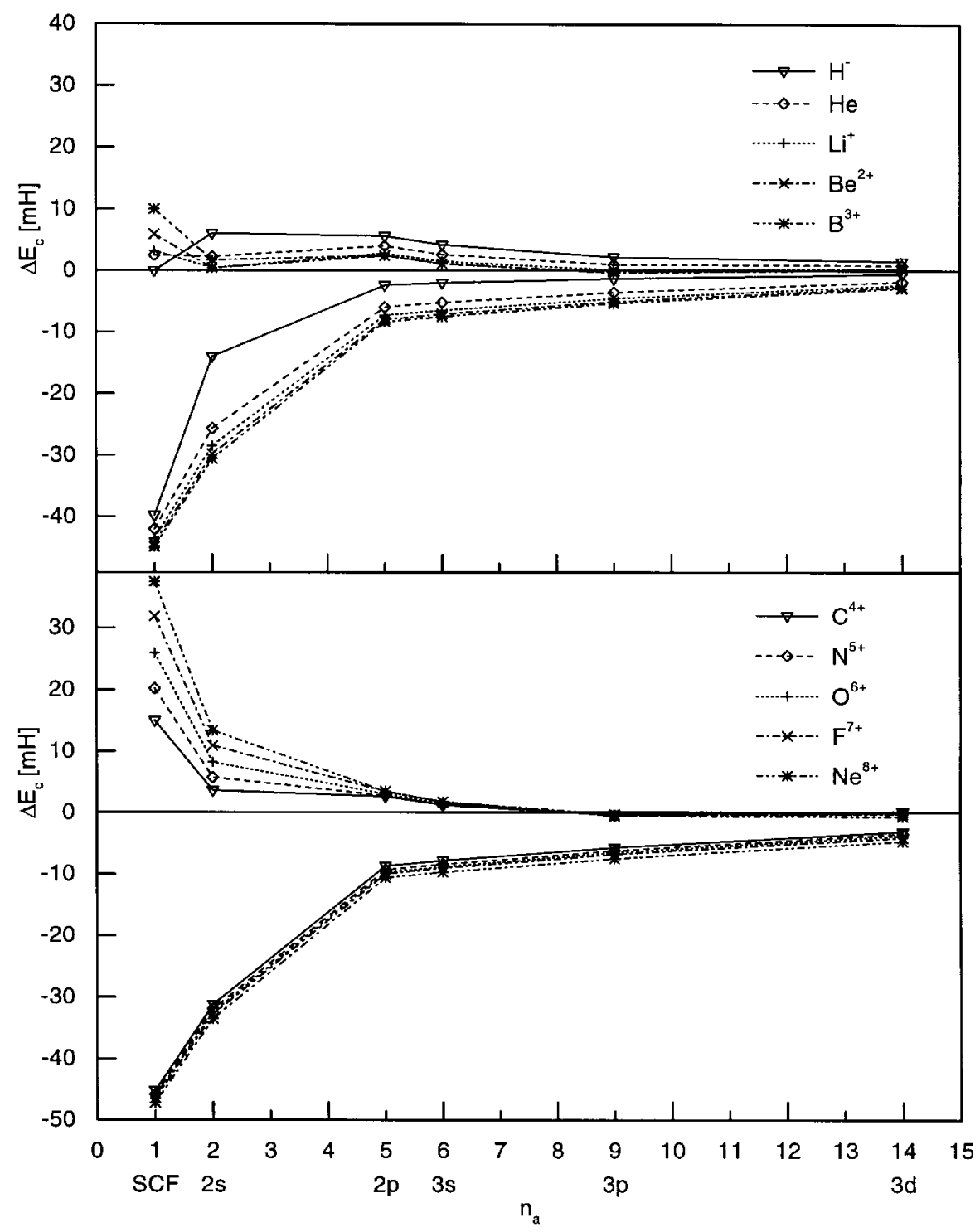

Figure 2. Deviations $\Delta E_{\mathrm{c}}$ of CASSCF (lower curves) and CASSCF-DFT (upper curves) results for two-electron ions $\mathrm{X}^{(Z-2)+}$ from 'experimental' correlation energies, for various definitions of the active space $\left(n_{\mathrm{a}}\right.$ is the number of active orbitals; $n_{\mathrm{a}}=1$ corresponds to the standard SCF and (gradient-corrected exact-exchange) DFT result).

\subsection{The Be isoelectronic series}

In contrast with the He series, the $X^{(Z-4)+}$ ions $(X=\mathrm{Li}, \mathrm{Be}, \mathrm{B}, \ldots)$ are characterized by an important non-dynamical $\mathrm{s}^{2} \rightarrow \mathrm{p}^{2}$ correlation contribution which linearly scales with $Z[18]$ the LDA correlation energies which increase as $\ln Z$ only, badly underestimate $\left|E_{\mathrm{c}}\right|$ for large $Z$ (by about $40 \mathrm{mH}$ for $\mathrm{Ne}^{6+}$ ).

In our calculations, we used again large uncontracted basis sets, of the size 13s10p6d (15s10p6d for $\left.\mathrm{Li}^{-}\right)$, with s exponents taken from van Duijneveldt [20] and Poirier et al. [21] supplemented by diffuse exponents 0.00985 and 0.0043183 in the case of $\mathrm{Li}^{-}$), and even-tempered p, d sets (scaling factors 2.5 and centres 1.0 and 2.5 for $\mathrm{Be}$, with rescaling for other atoms as described in the last subsection). CASSCF calculations were performed, using these basis sets, for the following active spaces: $2 \mathrm{~s}$, $2 \mathrm{~s} 1 \mathrm{p}, 3 \mathrm{~s} 1 \mathrm{p}, 3 \mathrm{~s} 2 \mathrm{p}, 4 \mathrm{~s} 2 \mathrm{p}, 4 \mathrm{~s} 2 \mathrm{p} 1 \mathrm{~d}$, which are ordered according to decreasing energy gain per orbital. (An exception is $\mathrm{Li}^{-}$, where the sequence begins with $2 \mathrm{~s}, 3 \mathrm{~s}, 3 \mathrm{~s} 1 \mathrm{p}, \ldots$...) DFT contributions are evaluated in the same way as specified for the He series.

Figure 6 shows results for correlation-energy errors, at both the CASSCF and the CASSCF-DFT levels. The SCF errors are much larger than for the He series $\left(180 \mathrm{mH}\right.$ for $\mathrm{Ne}^{6+}$ as against $50 \mathrm{mH}$ for $\mathrm{Ne}^{8+}$ ), since not only non-dynamical contributions but also core correlation energies now have to be accounted for. At the SCF-DFT levels, the errors are much attenuated but 


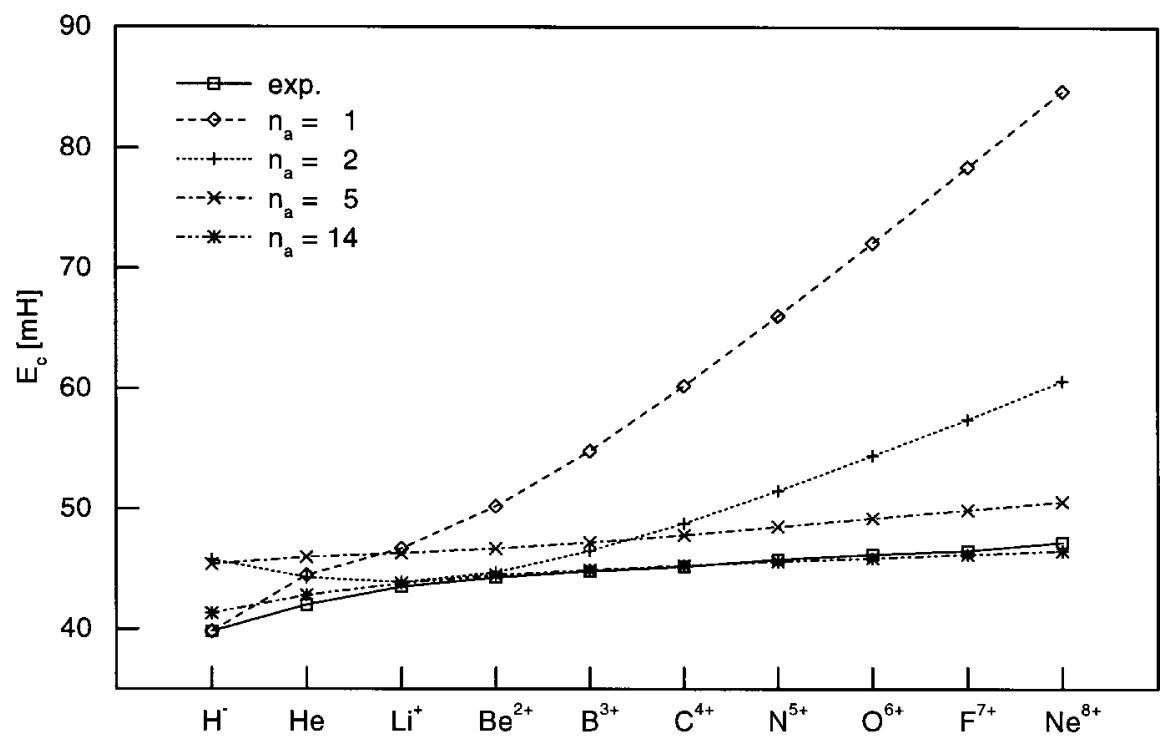

Figure 3. CASSCF-DFT correlation energies $E_{\mathrm{c}}$ for two-electron ions $\mathrm{X}^{(\mathrm{Z}-2)+}$ as a function of $Z$, in comparison with experimental results; $n_{\mathrm{a}}$ is the number of active orbitals included; a gradient-corrected density functional is used, cf. text.

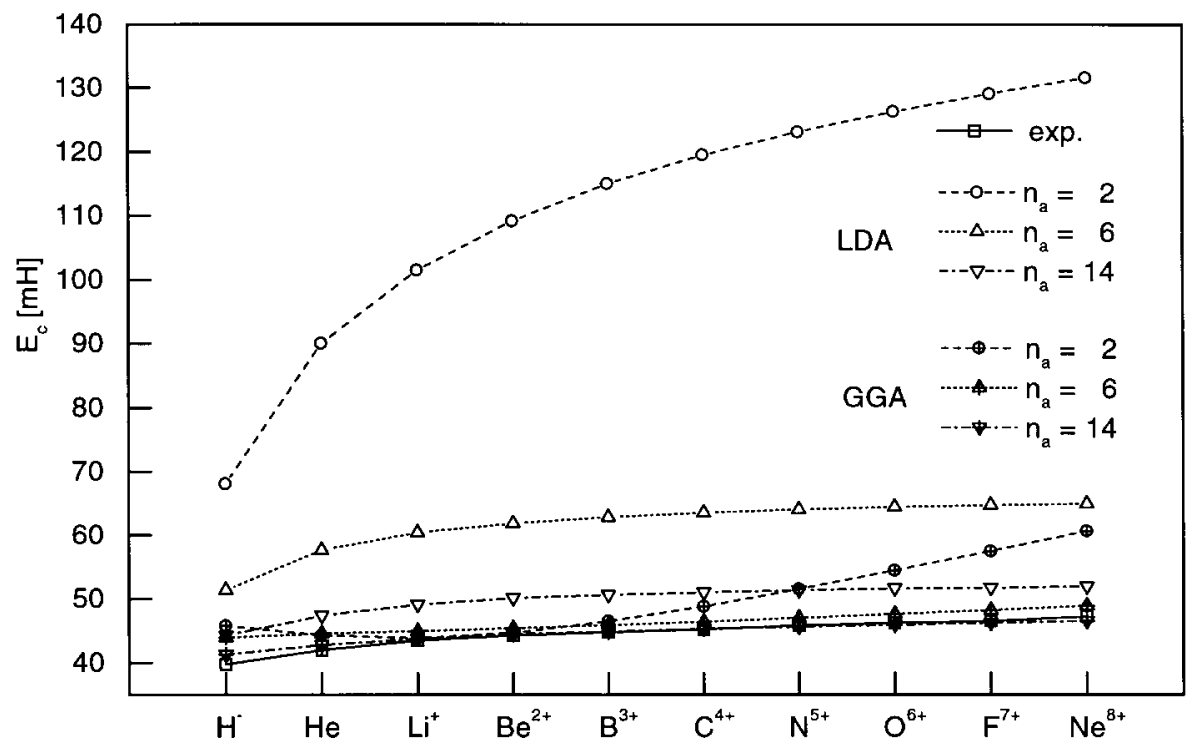

Figure 4. Comparison of CASSCF-DFT correlation energies $E_{\mathrm{c}}$ for two-electron ions $\mathrm{X}^{(\mathrm{Z}-2)+}$ from correlation-energy density functionals based on the LDA and including gradient corrections (GGA) respectively; $n_{\mathrm{a}}$ is the number of active orbitals.

still sizeable (about $45 \mathrm{mH}$ for $\mathrm{Ne}^{6+}$ ). Including the $2 \mathrm{~s}^{2} \rightarrow 2 \mathrm{p}^{2}$ quasidegeneracy ( $2 \mathrm{~s} 1 \mathrm{p}$ active space), the CASSCF-DFT results deviate by less than $24 \mathrm{mH}$ from the 'experimental' correlation energies, but the sign of the deviation changes (from underestimation to overestimation). The reason is the DFT overestimation of dynamical correlation energies already seen for the He series; in particular, the description of the $1 \mathrm{~s}^{2}$ core correlation energy is virtually at the SCF-DFT level still, with this active space. This can be remedied by including the next s orbital into the active space, yielding an improved accuracy of $7 \mathrm{mH}$ or less. Further extension of the active space leads to a uniform and monotonic convergence towards experiment.

For the $Z$ dependence of the CASSCF-DFT correlation energies, depicted in figure 7 , the same remark applies as for the He series; adding one s plus one $p$ orbital to the SCF space is sufficient for efficiently correcting the artificial $\ln Z \mathrm{SCF}$-DFT dependence, leading to a curve linear in $Z$, very close to the experimental 


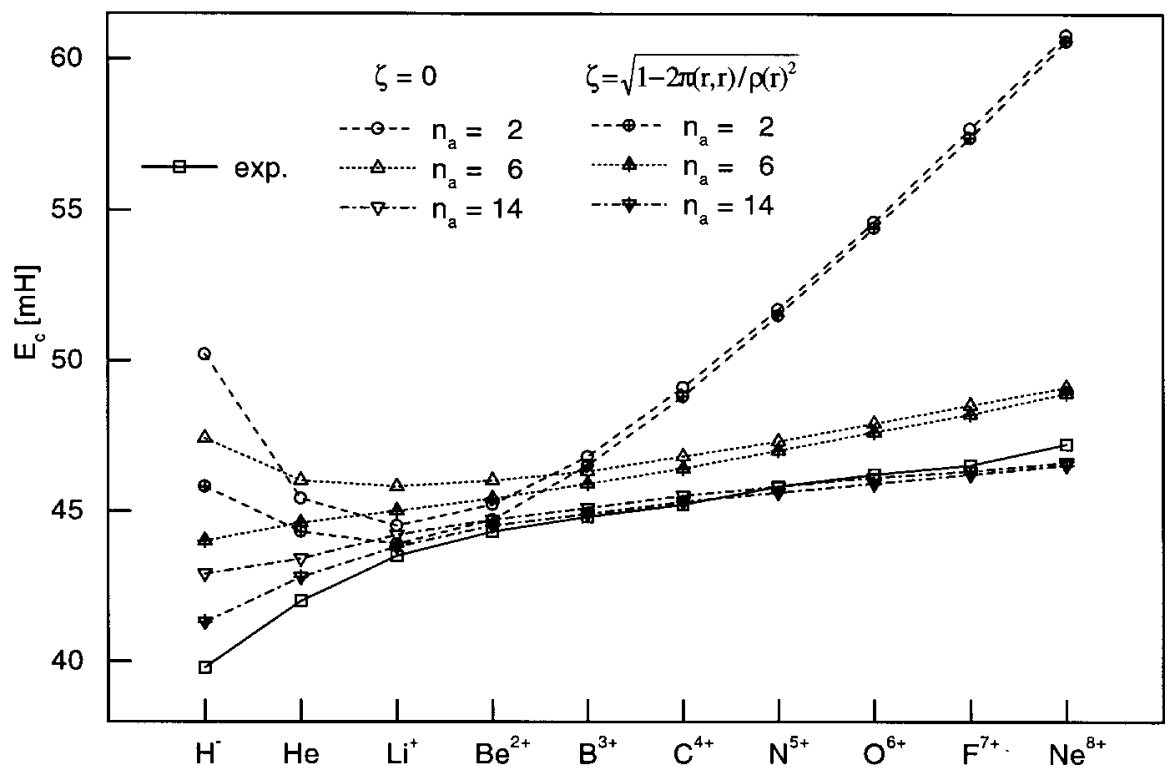

Figure 5. Comparison of CASSCF-DFT correlation energies $E_{\mathrm{c}}$ for two-electron ions $\mathrm{X}^{(\mathrm{Z}-2)+}$ from correlation-energy density functionals without and with inclusion of information on the pair density $\pi$ respectively (in the former case, a zero spin polarization $\zeta=0$ is used); $n_{\mathrm{a}}$ is the number of active orbitals.

Table 2. CASSCF and CASSCF-DFT spectroscopic constants for the $\mathrm{H}_{2}$ molecule, for various activespace sizes $n_{\mathrm{a}}\left(n_{\mathrm{a}}=1\right.$ corresponds to the standard SCF and (gradient-corrected exact-exchange) DFT result), in comparison with full $\mathrm{CI}$ and experimental results.

\begin{tabular}{cccccccc}
\hline & \multicolumn{3}{c}{ CASSCF } & & \multicolumn{3}{c}{ CASSCF-DFT } \\
\cline { 2 - 3 } \cline { 6 - 8 }$n_{\mathrm{a}}$ & $r_{\mathrm{e}} / \mathrm{au}$ & $D_{\mathrm{e}} / \mathrm{H}$ & $\omega_{\mathrm{e}} / \mathrm{cm}^{-1}$ & & $r_{\mathrm{e}} / \mathrm{au}$ & $D_{\mathrm{e}} / \mathrm{H}$ & $\omega_{\mathrm{e}} / \mathrm{cm}^{-1}$ \\
\hline 1 & $1 \cdot 387$ & $0 \cdot 1336$ & $4599 \cdot 9$ & & $1 \cdot 383$ & $0 \cdot 1806$ & $4636 \cdot 1$ \\
2 & $1 \cdot 426$ & $0 \cdot 1523$ & $4282 \cdot 4$ & & $1 \cdot 413$ & $0 \cdot 1811$ & $4399 \cdot 1$ \\
3 & $1 \cdot 415$ & $0 \cdot 1596$ & $4347 \cdot 4$ & & $1 \cdot 408$ & $0 \cdot 1789$ & $4417 \cdot 7$ \\
5 & $1 \cdot 405$ & $0 \cdot 1700$ & $4415 \cdot 4$ & & $1 \cdot 403$ & $0 \cdot 1804$ & $4445 \cdot 2$ \\
6 & $1 \cdot 405$ & $0 \cdot 1706$ & $4418 \cdot 3$ & & $1 \cdot 403$ & $0 \cdot 1790$ & $4439 \cdot 3$ \\
8 & $1 \cdot 405$ & $0 \cdot 1713$ & $4421 \cdot 4$ & & $1 \cdot 402$ & $0 \cdot 1777$ & $4442 \cdot 6$ \\
10 & $1 \cdot 404$ & $0 \cdot 1717$ & $4425 \cdot 7$ & & $1 \cdot 403$ & $0 \cdot 1771$ & $4443 \cdot 2$ \\
Full CI & $1 \cdot 402$ & $0 \cdot 1739$ & $4438 \cdot 2$ & & & & \\
Experimental & $1 \cdot 401$ & $0 \cdot 1745$ & $4400 \cdot 4$ & & & &
\end{tabular}

curve. (We note that the use of the two-particle on-top density for evaluating $\zeta$ (instead of setting $\zeta=0$ ) leads to a nearly constant shift $\left(7 \mathrm{mH}\right.$ for $\left.\mathrm{C}^{2+}\right)$ of the calculated curve, significantly improving agreement with experiment.)

Usually, total correlation energies are of less importance than differential correlation contributions to properties. Let us next consider, therefore, the performance of the CASSCF-DFT approach for the (first) ionization potential (IP) of the Be series. The IP values are derived from separate calculations for the (closedshell) $\mathrm{X}^{(Z-4)+}$ and the (open-shell) $\mathrm{X}^{(Z-3)+}$ ions. Al- though our approach presumably is less accurate for the latter than for the former, since the correction function $\varphi$ (equation (6)) has only been calculated for $\zeta=0$ and is used for $\zeta \neq 0$ without change, the results are quite encouraging (figure 8 ). With a $3 \mathrm{~s} 1 \mathrm{p}$ active space, the SCF-DFT IPs are greatly improved, again leading to the required linear $Z$ behaviour.

\subsection{The $\mathrm{H}_{2}$ molecule}

Potential curves of molecules with covalent bonds are very good candidates for the application of our new approach; near-degeneracy effects become very 


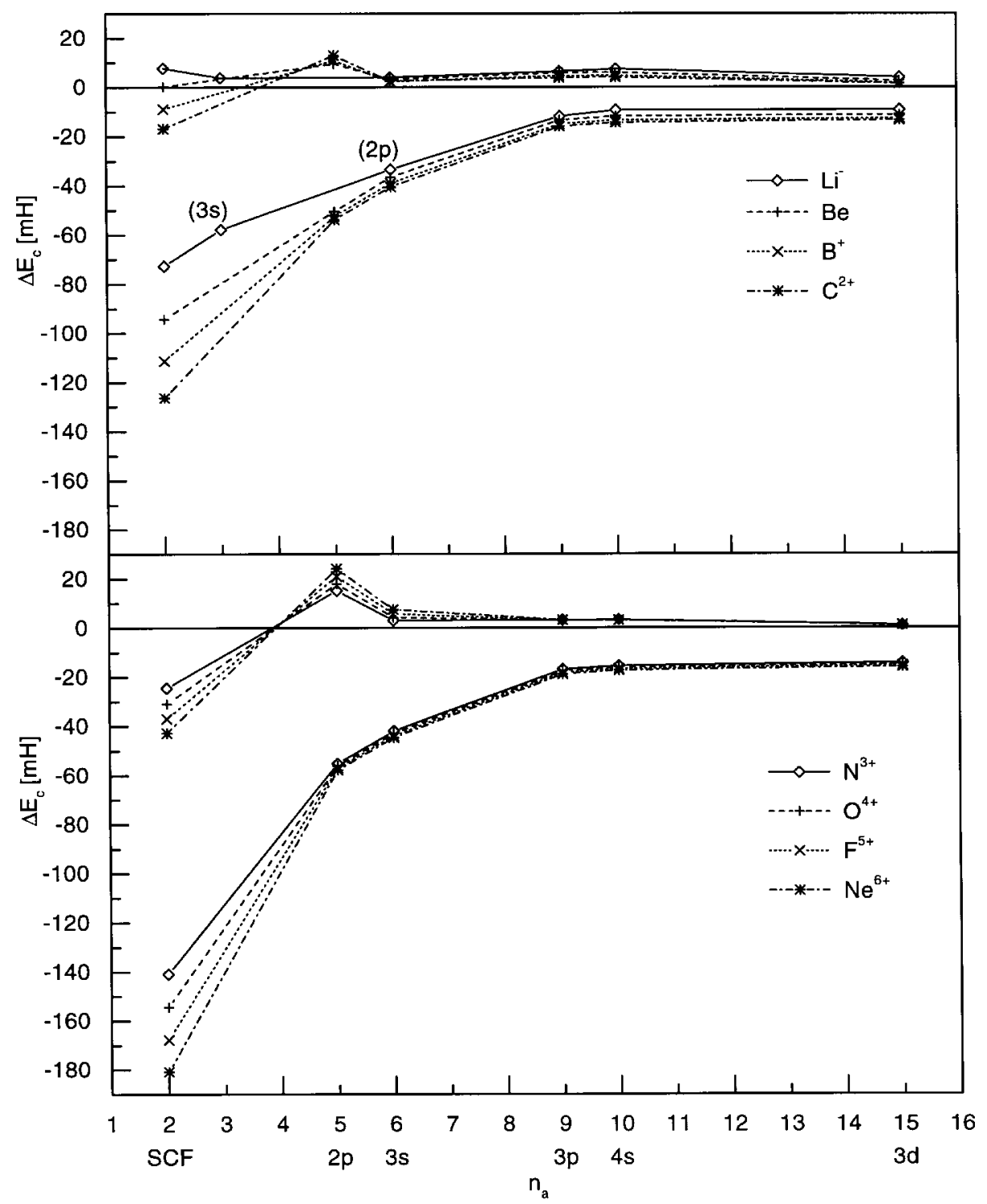

Figure 6. Deviations $\Delta E_{\mathrm{c}}$ of CASSCF (lower curves) and CASSCF-DFT (upper curves) results for four-electron ions $\mathrm{X}^{(Z-4)+}$ from 'experimental' correlation energies, for various definitions of the active space $\left(n_{\mathrm{a}}\right.$ is the number of active orbitals; $n_{\mathrm{a}}=2$ corresponds to the standard SCF and (gradient-corrected exact-exchange) DFT result).

strong at the dissociation limit, and SCF-DFT is bound to fail here, since DFT cannot compensate the large Hartree-Fock error for $r \gg r_{\mathrm{e}}$ without symmetry breaking.

Let us first consider the $\mathrm{H}_{2}$ potential curve, as the simplest example. We used the $10 \mathrm{~s} 8 \mathrm{p} 5 \mathrm{~d}$ basis set described for $\mathrm{H}^{-}$in section 3.1. The following orbitals were successively included into the active space: $1 \sigma_{\mathrm{g}}$, $1 \sigma_{\mathrm{u}}, 2 \sigma_{\mathrm{g}}, 1 \pi_{\mathrm{u}}, 2 \sigma_{\mathrm{u}}, 1 \pi_{\mathrm{g}}, 1 \delta_{\mathrm{g}}$. Again the sequence was chosen according to decreasing CASSCF energy contributions. Results for equilibrium bond length $r_{\mathrm{e}}$, dissociation energy $D_{\mathrm{e}}$ and vibrational frequency $\omega_{\mathrm{e}}$ are given in table 2. It is seen that the CASSCF-DFT results constitute a significant improvement over CASSCF only. Of course, with only one orbital in the active space ( $\mathrm{SCF}-\mathrm{DFT}$ ), the potential curve is incorrect in shape (leading to a too small $r_{\mathrm{e}}$ and too large $\omega_{\mathrm{e}}$ ), and the dissociation energy is only acceptable since the exact (non-relativistic) energy of two separated $\mathrm{H}$ atoms was taken as reference. However, already with a minimal active space allowing for proper dissociation $\left(1 \sigma_{\mathrm{g}}, 1 \sigma_{\mathrm{u}}\right)$, the potential curve is very similar to the exact curve (figure 9): $r_{\mathrm{e}}$ is accurate to $1 \mathrm{pm}$ and the dissociation energy is off by $7 \mathrm{mH}$ only. When $D_{\mathrm{e}}$ is evaluated with respect to the molecular result at large distances $(r=10$ Bohr) instead of that for (exact) separated atoms, the atomic DFT error virtually cancels out, and the error in the dissociation energy with a twoorbital active space is reduced to $1.8 \mathrm{mH}$. 


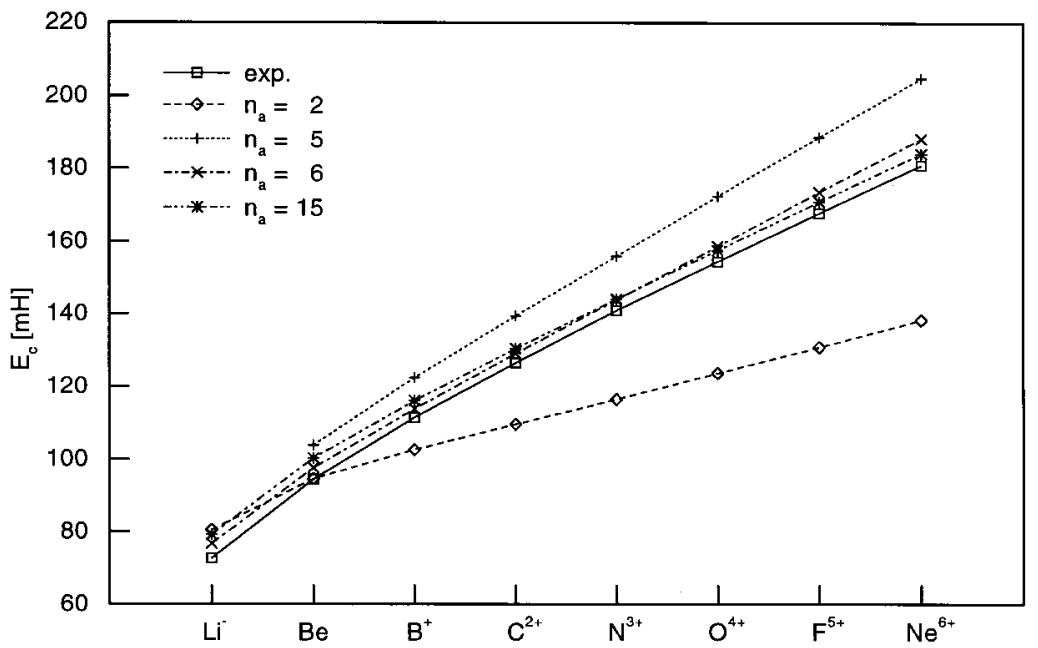

Figure 7. CASSCF-DFT correlation energies $E_{\mathrm{c}}$ for four-electron ions $\mathrm{X}^{(Z-4)+}$ as a function of $Z$, in comparison with experimental results; $n_{\mathrm{a}}$ is the number of active orbitals included; a gradient-corrected density functional is used, cf. text.

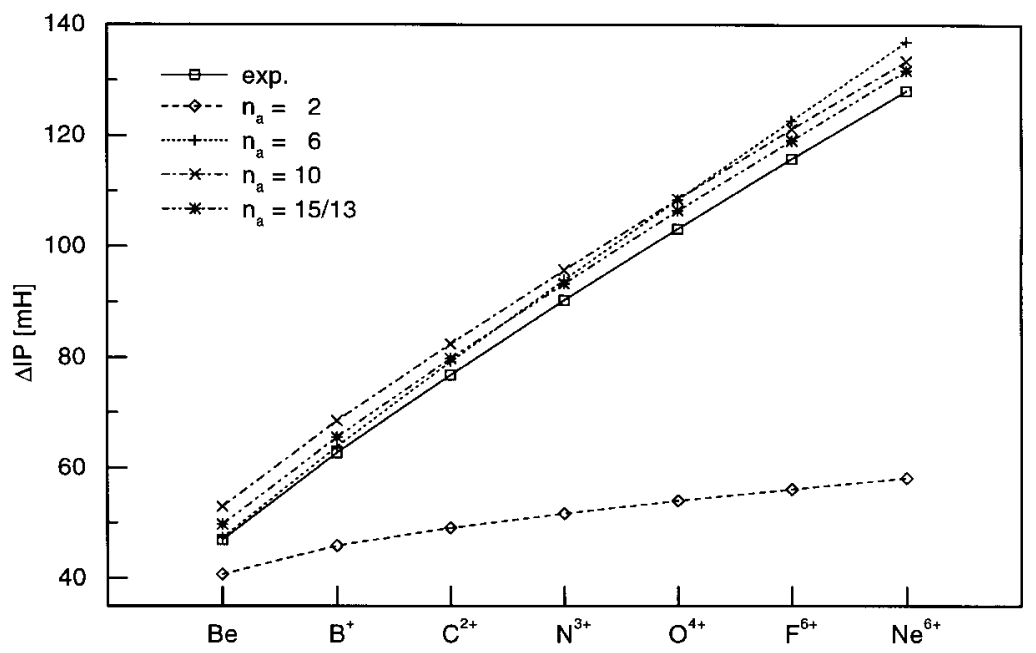

Figure 8. CASSCF-DFT correlation contribution to the IPs of the four-electron ions $\mathrm{X}^{(Z-4)+} ; n_{\mathrm{a}}$ is the number of active orbitals included; a gradient-corrected density functional is used, cf. text.

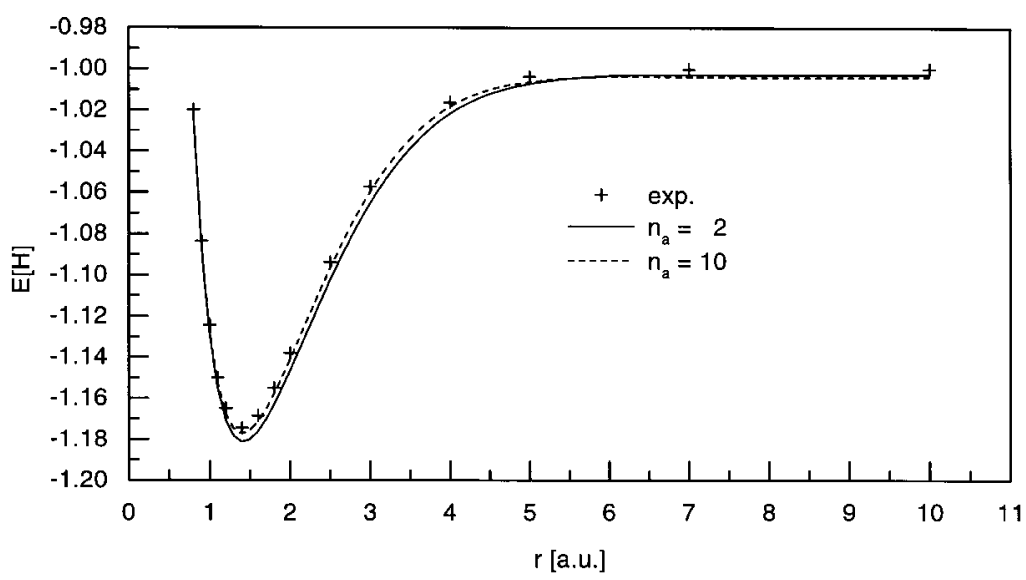

Figure 9. CASSCF-DFT potential curve of the $\mathrm{H}_{2}$ molecule, for two active spaces $\left(n_{\mathrm{a}}\right.$ is the number of active orbitals), in comparison with experimental points; a gradient-corrected density functional is used, cf. text. 


\section{Conclusions}

We have described a modification of usual gradientcorrected correlation-energy density functionals, so that they can be used in connection with CASSCF wavefunctions. Drawing information from the on-top pair density, in addition to the one-particle density, resolves problems of symmetry breaking. Introducing a correction factor, depending on the active space employed, enables a smooth transition between the limiting case of a pure density-functional and a full-CI calculation.

We have shown, for the correlation contributions to the ionization energies of two- and four-electron ions and the potential curve of $\mathrm{H}_{2}$, that DFT errors can be effectively removed while still keeping the active-space size relatively small.

The authors are indebted to Professor H.-J. Werner (Stuttgart) for making available his program package Molpro.

\section{References}

[1] Nalewajski, R. F. (editor), 1996, Density Functional Theory, Vols. I-IV, Topics in Current Chemistry, Vols. 180-183. (Berlin: Springer).

[2] Yarkony, D. R. (editor), 1995, Modern Electronic Structure Theory, Parts I and II, Advanced Series in Physical Chemistry, Vol. 2 (Singapore: World Scientific).

[3] Savin, A., 1991, Density Functional Methods in Chemistry, edited by J. K. Labanowski and J. W. Andzelm (Berlin: Springer), p. 213.

[4] Becke, A. D., Savin, A., and Stoll, H., 1995, Theor. chim. Acta, 91, 147.

[5] Perdew, J. P., Savin, A., and Burke, K., 1995, Phys. Rev. A, 51, 4531.

[6] Moscardo, F., and San-Fabian, E., 1991, Phys. Rev. A, 44, 1549.
[7] Stoll, H., and Savin, A., 1985, Density Functional Methods in Physics, edited by R. M. Dreizler and J. da Providencia (New York: Plenum), p. 177.

[8] Savin, A., and Flad, H.-J., 1995, Int. J. quant. Chem., 56, 327.

[9] Savin, A., 1988, Int. J. quantum Chem. S, 22, 59.

[10] Savin, A., 1996, Recent Advances in Density Functional Methods, edited by D. P. Chong (Singapore: World Scientific), p. 129.

[11] Mienlich, B., 1990, Diplomarbeit, Universität Stuttgart.

[12] Ceperley, D. M., and Alder, B. J., 1980, Phys. Rev. Lett., 45, 566.

[13] Vosko, S. H., Wilk, L., and Nusair, M., 1980, Can. J. Phys., 58, 1200.

[14] Perdew, J. P., 1986, Phys. Rev. B, 33, 8822.

[15] Levy, M., 1979, Proc. natl. Acad. Sci. USA, 76, 6062.

[16] Savin, A., Stoll, H., and Preuss, H., 1986, Theor. chim. Acta, 70, 407.

[17] Mienlich, B., Savin, A., Stoll, H., and Preuss, H., 1989, Chem. Phys. Lett., 157, 200.

[18] Linderberg, J., and Shull, H., 1960, J. molec. Spectrosc., 5, 1 .

[19] Perdew, J. P., McMullen, E. R., and Zunger, A., 1981, Phys. Rev. A, 23, 2785.

[20] van Duijneveldt, F. B., 1971, IBM Research Report No. 945.

[21] Poirier, R., Kari, R., and Csizmadia, I. G., 1985, Handbook of Gaussian Basis Sets (Amsterdam: Elsevier).

[22] Almlöf, J., and Taylor, P. R., 1987, J. chem. Phys., 86, 4070.

[23] Werner, H.-J., and Knowles, P. J., with contributions from Almlöf, J., Amos, R. D., Berning, A., Deegan, M. J. O., Eckert, F., Elbert, S. T., Hampel, C., Lindh, R., Meyer, W., Nicklass, A., Peterson, K., Pitzer, R., Stone, A. J., Taylor, P. R., Mura, M. E., Pulay, P., Schuetz, M., Stoll, H., Thorsteinsson, T., and Cooper, D. L., 1994, Molpro a Package of ab initio Programs.

[24] Werner, H.-J., and Knowles, P. J., 1985, J. chem. Phys., 82, 5053.

[25] Knowles, P. J., and Werner, H.-J., 1985, Chem. Phys. Lett., 115, 259. 